\title{
Increased NCAM Expression and Vascular Development in Trisomy 16 Mouse Embryos: Relationship with Nuchal Translucency
}

\author{
MIREILLE N. BEKKER, JENNY B. ARKESTEIJN, NYNKE M.S. VAN DEN AKKER, STANLEY HOFFMAN, \\ SANDRA WEBB, JOHN M.G. VAN VUGT, AND ADRIANA C. GITTENBERGER-DE GROOT
}

\begin{abstract}
Department of Obstetrics and Gynecology [M.N.B., J.M.G.V.], VU University Medical Center, 1081 HV, Amsterdam, the Netherlands; Department of Anatomy and Embryology [M.N.B., J.B.A., N.M.S.A., A.C.G.G.],

Leiden University Medical Center, 2300 RC, Leiden, the Netherlands; Department of Cell Biology [S.H.], Medical University of South Carolina, Charleston, SC 29425; Department of Basic Medical Sciences, Anatomy and Developmental Biology [S.W.], St. George Hospital Medical School, London, SW17 ORE, U.K.
\end{abstract}

\begin{abstract}
Increased nuchal translucency in the human fetus is associated with chromosomal abnormalities, enlarged jugular lymphatic sacs, cardiac defects and changed flow through the ductus venosus. The developmental background of this nuchal edema in relation to the associated anomalies remains elusive. We studied the morphologic correlation between neurogenesis and vasculogenesis in neck, heart, and ductus venosus region of wild type and trisomy 16 mice embryos (E10- E18), using an antibody against Neural Cell Adhesion Molecule (NCAM). Trisomy 16 mice are a model for the above described human phenotype. From E12 trisomy 16 mice showed an altered arrangement of cranial nerves IX, X and XI, which are positioned between the carotid artery, jugular vein and enlarged lymphatic sac. The vagal nerve was significantly smaller, compared with wild type embryos. NCAM was over expressed in both neuronal and cardiovascular structures in trisomy 16 mice, being particularly prominent in the $4^{\text {th }}$ and $6^{\text {th }}$ pharyngeal arch arteries, and the
\end{abstract}

\section{ABSTRACT}

ductus venosus. In the $4^{\text {th }}$ and $6^{\text {th }}$ pharyngeal arch arteries, NCAM over expression was located to the part of the vessel wall that is closely related to the vagal and recurrent nerve. In case of $4^{\text {th }}$ pharyngeal arch artery abnormalities NCAM expression, on the other hand, was reduced. In conclusion, the interaction between neurogenesis and vasculogenesis is disturbed in the trisomy 16 mouse model, and might be a common denominator in the spectrum of anomalies associated with increased nuchal translucency. (Pediatr Res 58: 1222-1227, 2005)
JLS, jugular lymphatic sac
Abbreviations
NCAM, Neural Cell Adhesion Molecule
NT, nuchal translucency
PAA, pharyngeal arch artery
RUNX1, Runt-related gene-1

Ultrasonographic measurement of nuchal translucency (NT) in the human fetus at gestational age between 10 to $14 \mathrm{wk}$ is a common screening method to detect trisomy 21 . Increased NT is associated with a spectrum of anomalies, including aneuploidy, isolated cardiac defects, and changed flow velocities through the ductus venosus $(1,2)$.

The developmental background of increased NT and associated findings is still insufficiently understood. Recently, a disturbed lymphangiogenesis has been suggested as a basis for the nuchal edema because of the concomitant abnormal enlargement and persistence of the jugular lymphatic sacs (JLS) in both human fetuses and mouse embryos with

Received February 2, 2005; accepted April 29, 2005.

Correspondence: Adriana C. Gittenberger-de Groot, Ph.D., Department of Anatomy and Embryology, Leiden University Medical Center, PO Box 9602, 2300RC Leiden, the Netherlands; email: acgittenlumc.nl

DOI: 10.1203/01.pdr.0000187795.82497.31 nuchal edema $(3,4)$. This theory explains both the regional accumulation of the fluid in the neck region and the temporary character of the NT enlargement. Other suggested theories, like temporary cardiac failure $(5,6)$ and the alteration of extracellular matrix components (7) might be part of the causal pathway, but fail to completely elucidate this phenomenon.

A study (3) of trisomy 16 embryos showed that the endothelium of the enlarged JLS was abnormally thickened. Also, there was a very close correlation of nerves with the JLS and other vascular structures. A mutual influence of nerves and endothelium could play a role in the development of nuchal translucency, since vessels and nerves are known to share many developmental genes (8).

The hypothesis for this study was that a disturbance in the interaction between neurogenesis and vasculogenesis is a common denominator in the spectrum of associated (lymphatic) 
vascular and cardiovascular abnormalities in fetuses with increased NT.

Innervation and vascular morphology in neck, heart, and ductus venosus region of wild type and trisomy 16 mouse embryos were investigated. Trisomy 16 mice embryos have nuchal edema, enlarged JLS and cardiac defects. Also, the trisomy 16 mouse is proposed as an animal model for human trisomy 21 as the mouse chromosome 16 contains the syntenic region for the human chromosome band 21q22 (9).

In this model we studied the expression of Neural Cell Adhesion Molecule (NCAM) because it is expressed both in nerves and the cardiovascular system (10). NCAM is of special interest, as this protein is regulated by Runt-related gene-1 (RUNX1), which is described as triplicated gene in the trisomy 16 mouse and human trisomy $21(11,12)$.

\section{METHODS}

Embryos. Wild type and trisomy 16 mouse embryos from gestational age day 10 (E10) to E18 d (Table 1) were collected (13). The study was approved by the Animal Care and Use Committee of St. George Hospital, London. The embryos were fixed in $4 \%$ paraformaldehyde at $4^{\circ} \mathrm{C}$ overnight. After dehydrating the embryos with xylene, they were embedded in paraffin and serially sectioned $(5 \mu \mathrm{m})$. The sections were alternately mounted on 5 subsequent slides, so that 5 different staining procedures could be performed.

Immunohistochemistry. Consecutive sections were stained with an NCAM antibody. Using an antibody against NCAM (provided by S. Hoffman, Charleston, S.C., U.S.A.) in an immunohistochemical staining all cells derived from neural tissue are stained.

First, the slides were deparaffinated. Between all steps the slides were rinsed twice with phospate-buffered saline (PBS) and once with PBS/0.05\% Tween unless indicated otherwise. Before staining, the slides were microwaveprocessed by heating them 3 times for $4 \mathrm{~min}$ to $99^{\circ} \mathrm{C}$ in a citric acid buffer $(0.01 \mathrm{M}$ in distilled water, $\mathrm{pH}=6.0)$. Then the slides were rinsed twice with PBS and incubated for 15 min in a $0.3 \% \mathrm{H}_{2} \mathrm{O}_{2}$ solution to block the endogenous peroxidase activity. Subsequently, the slides were incubated overnight with the specific antibody against NCAM. After that, the sections were incubated for 90 min with the second antibody, GAR-Ig. The third antibody used was R-PAP, with which the slides were incubated for 90 min. Afterward, the slides were rinsed twice with PBS and once with Tris/Maleate ( $\mathrm{pH}$ 7.6). As chromogen, DAB (3-3'diaminobenzidin tetrahydrochloride) was used. A counter staining was performed by dipping the slides for $10 \mathrm{~s}$ in Mayer's haematoxylin. The slides were rinsed for $10 \mathrm{~min}$ in tap water, dehydrated to xylene and coated before attaching a coverslip.

Morphometric analysis. The volume of the vagal nerve was measured in three wild type and three trisomy 16 mouse embryos of E14. Morphometry of the vagal nerve was performed because of its important role and localization in nuchal, heart and ductus venosus region.

From each embryo, in 25 slides with an equal interval of $75 \mu \mathrm{m}$ the surface of the vagal nerve was measured systematically, as proposed by Bouman et al. (14). Also, in each slide the area of NCAM positivity in the vagal nerve was measured. The first slide was at the level of caudal end of the eye. The last slide was at the level of the ductus arteriosus. The exact position of the last slide differed slightly, according to the overall size of each embryo. The combined volume of the vagal nerve and the NCAM expressing area were calculated by

Table 1. Number of mouse embryos examined per embryonic day

\begin{tabular}{ccc}
\hline Embryonic day & Wild type (no.) & Trisomy 16 (no.) \\
\hline 10 & 3 & 2 \\
11 & 2 & 1 \\
12 & 3 & 2 \\
13 & 2 & 1 \\
14 & 4 & 3 \\
15 & 2 & 3 \\
16 & 1 & 2 \\
17 & 2 & 1 \\
18 & 1 & 1 \\
\hline
\end{tabular}

the method described by Cavalieri (15). The student's $t$ test was used to compare the volumes. Significance was set on $p<0.05$.

\section{RESULTS}

\section{Neck Region}

Wild type. At E10, no positive cells could be observed in NCAM stained sections. From E12 on, there was a grouping of the JLS, the jugular vein, the carotid artery and three cranial nerves $(\mathrm{CN})$ : CN IX (glossopharyngeal nerve), $\mathrm{CN} X$ (vagal nerve) and CN XI (accessory nerve). The carotid artery was positioned medially and ventrally to the jugular vein. The JLS was located lateral and dorsally. The cranial nerves lay grouped together between the jugular vein, carotid artery and JLS (Fig. 1A). The JLS was crossed by cervical nerves III, IV and $\mathrm{V}$.

Trisomy 16. At E10-11, there was no difference in NCAM staining or morphology compared with wild type embryos. At E12, the JLS was enlarged and in the nuchal edema was observed. From E12 on, a consistent grouping of JLS, carotid artery, jugular vein and cranial nerves was not present, in

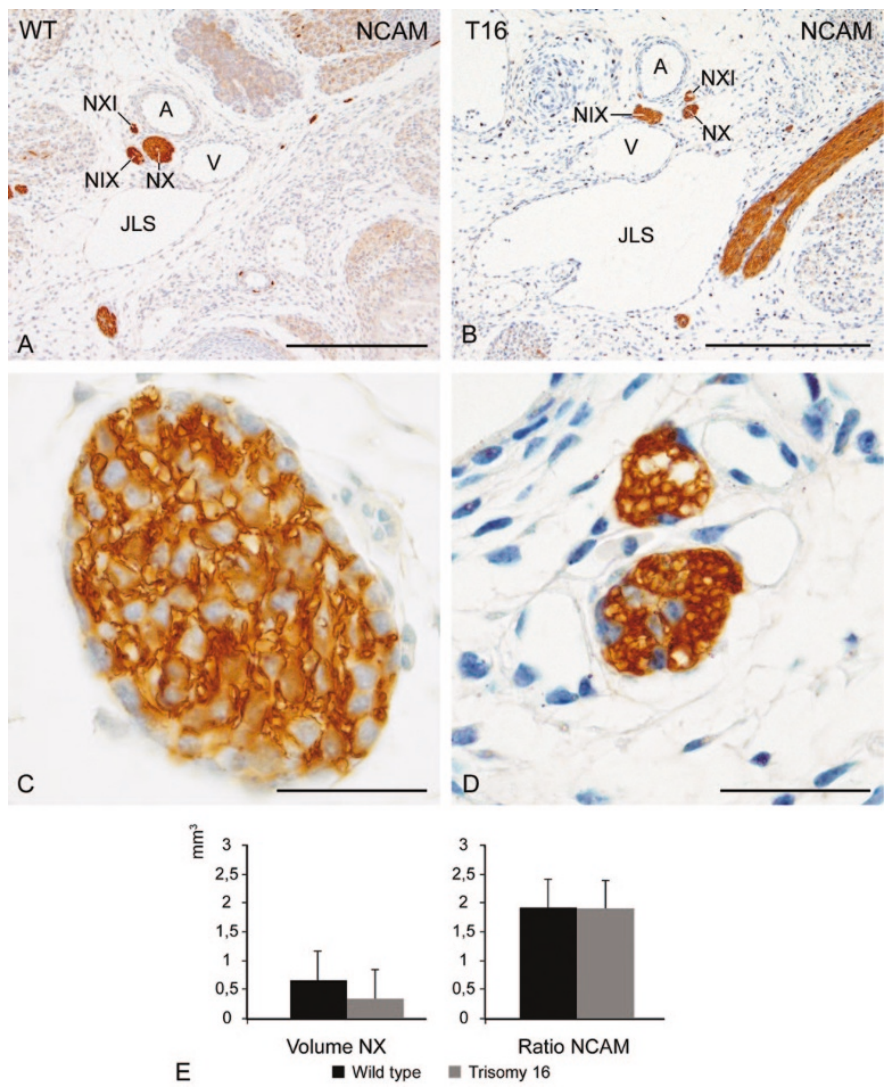

Figure 1. Transverse sections of the neck region of wild type (WT) and trisomy 16 (T16) mice at E14; stained for NCAM. (A) Grouping of cranial nerves IX, $X$ and XI between carotid artery (A), jugular vein $(V)$ and jugular lymphatic sac (JLS) in a WT mouse. (B) Different position of cranial nerves between carotid artery, jugular vein and enlarged jugular lymphatic sac in a T16 mouse. $(C)$ Detail of A: NX. $(D)$ Detail of B: NX. The NX is smaller and has an increased NCAM staining. Note the similar distribution of NCAM positivity and negativity. (E) Volume of the NX (left) and the ratio of the volume of NCAM positivity/negativity (right) between WT and T16 mice. The vagal was significantly smaller $(p<0.05)$ in T16 mice. Abbreviations: NX vagal nerve; NIX glossopharyngeal nerve; NXI accessory nerve. Bars $=(A, C)$ $250 \mu \mathrm{m} ;(B, D) 25 \mu \mathrm{m}$. 
contrast to the wild type embryos. The cranial nerves were smaller and not in close proximity to one another (Fig. 1B).

Morphometric analysis of the vagal nerve revealed that the volume of the vagal nerve was significantly smaller in trisomy 16 mouse embryos $(p=0.028)$. The mean volume of the vagal nerve was $0.67 \pm 0.10 \mathrm{~mm}^{3}$ in wild type and $0.35 \pm 0.08 \mathrm{~mm}^{3}$ in trisomy 16 mouse embryos (Fig. 1E). Furthermore, no difference in the volume of NCAM positive tissue (neural cells) compared with the volume of the NCAM negative tissue (nuclei and fibroblasts) in the vagal nerve, was found between wild type and trisomy 16 mice embryos. This indicates that we are dealing with an overall smaller nerve (Fig. 1E). In addition, the immunostaining with NCAM did not allow identification of the precise anatomical components of the nerve as both neuronal cells, e.g., axons, and glial cells are NCAM positive.

The smaller nerves in the trisomy 16 embryos seemed to be more positive for NCAM, compared with normal littermates (Fig. 1C and $1 D$ ), but we could not substantiate this morphometrically. In the trisomy 16 embryos cervical nerves $\mathrm{I}$ to $\mathrm{V}$ passed through the enlarged JLS.

\section{Aortic Arch and Heart Region}

Wild type. From E12 on there was prominent NCAM expression in both $4^{\text {th }}$ and $6^{\text {th }}$ (left) pharyngeal arch arteries (PAA). In both $4^{\text {th }}$ PAA NCAM was expressed in the adventitia with extension toward the outer media in close proximity to the vagal and the recurrent nerve (Fig. 2E,I and Fig. 3D, G). The $6^{\text {th }}$ PAA stained positive in a small and specific region in the media on both sides of the vessel wall, also located in close apposition to the vagal and the recurrent nerve (Fig. 4A,B).

The myocardium bordering the site of semilunar valve and atrioventricular valve development was positive for NCAM on E12-14 (Fig. 4C), becoming more negative in older embryos (E15-E18). The aortico-pulmonary septum was positive (E12E18). The interventricular septum and remaining myocardium were negative (data not shown).

Trisomy 16. All trisomy 16 embryos showed septal defects ranging from complete atrioventricular septal to subaortic ventricular septal defects. A number of the trisomy 16 mouse embryos (9/16) showed a variable degree of $4^{\text {th }}$ PAA defects including hypoplasia, atresia and interruption. An interrupted right $4^{\text {th }}$ PAA was accompanied by a persistence of the right dorsal aorta and an aberrant subclavian artery. A cervical aortic arch was combined with interruption of the left fourth PAA. The combination of an aberrant subclavian artery or cervical aortic arch with interruption of the right or left $4^{\text {th }}$ PAA respectively, was observed in several embryos.

These $4^{\text {th }}$ PAA malformations coincided with outflow tract anomalies as double outlet right ventricle. The semilunar valves were dysplastic in some of the embryos. Septal and PAA defects were observed from E13 onwards.

A strong over expression of NCAM was observed in both $4^{\text {th }}$ and $6^{\text {th }}$ PAA in trisomy 16 embryos with normal PAA. The NCAM expressing region in the $4^{\text {th }} \mathrm{PAA}$, in proximity to the vagal and recurrent nerve, was similar as in wild type littermates but a larger area and higher intensity of staining in the tunica media was observed (Fig. $2 F, J$ and Fig. 3E,H). The
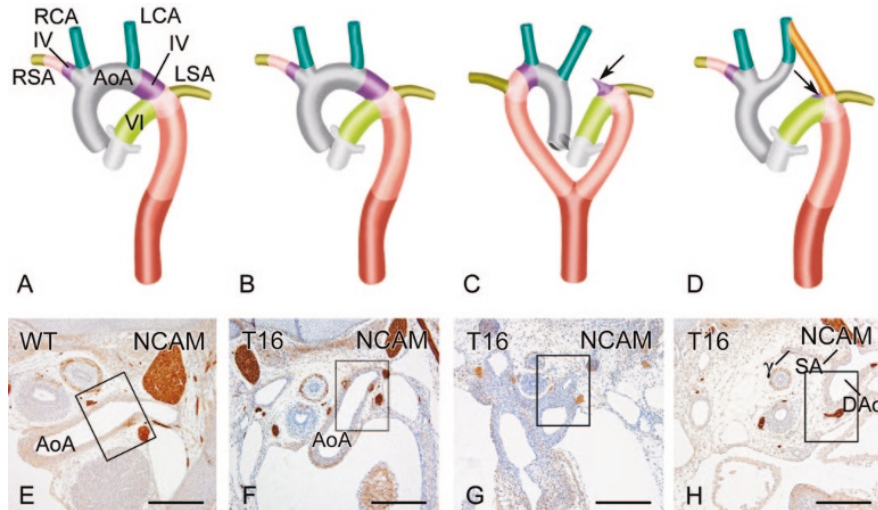

C

D
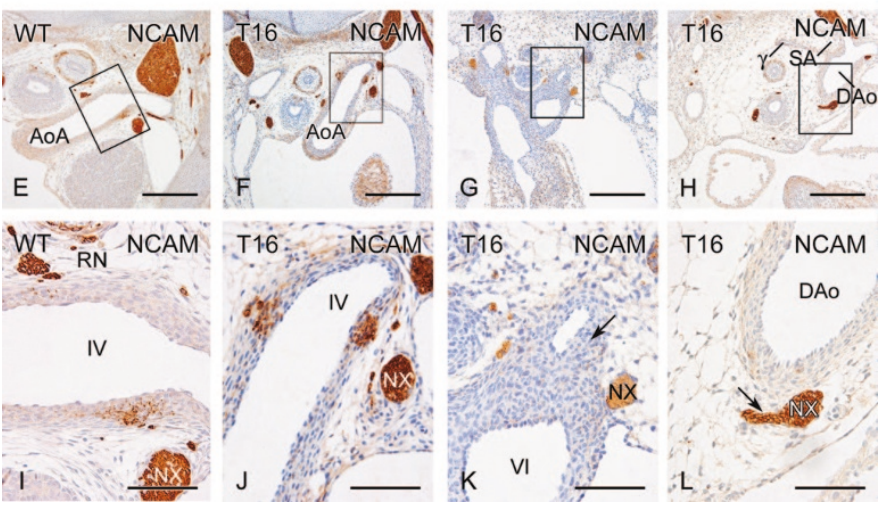

Figure 2. Transverse sections of the aortic arch (AoA) region wild type (WT) and trisomy 16 (T16) mice at E14; stained for NCAM or HE. (A-D) Schematic outline of normal PAA configuration; (A-B) hypoplastic left $(C)$ and interrupted left $4^{\text {th }}$ PAA $(D)$. Colors refer to distinct embryonic origins. $(E, I)$ Left $4^{\text {th }}$ PAA of a WT mouse with NCAM expression in the adventitia with extension into the media in close proximity to the vagal nerve (NX) and recurrent nerve $(\mathrm{RN})(F, J)$ Left $4^{\text {th }}$ PAA of a T16 mouse with a more intense and widespread vascular NCAM expression into the media. $(G, K)$ Diminished NCAM expression in the vessel wall of a hypoplastic $4^{\text {th }}$ PAA with an atretic end in a T16 mouse. The asterisk $(K)$ indicates the part of the vessel reflecting the $4^{\text {th }}$ PAA. The trans section is made just on the border between the ductus arteriosus and the atretic end. $(H, L)$ Solely NCAM expression in the NX in a T16 mouse with an interrupted $4^{\text {th }}$ PAA. The asterisk is indicating, where the $4^{\text {th }}$ PAA should have been positioned. Abbreviations: RSA/ LSA right/left subclavian artery; RCA/LCA right/ left carotid artery; DAo dorsal aorta; $\gamma$ $\gamma$-segment. Bars $=(E-H) 250 \mu \mathrm{m}$ and $(I-P) 50 \mu \mathrm{m}$.

ductus arteriosus (left $6^{\text {th }}$ PAA) was extremely positive and the expression area was broader compared with the wild type (Fig. $4 D, E)$.

Trisomy 16 embryos with $4^{\text {th }}$ PAA defects showed a different NCAM expression pattern. Those with a hypoplastic $4^{\text {th }}$ PAA showed a small number of NCAM expressing cells in the adventitia (Fig. $2 G, K$ ). In a case with an absent $4^{\text {th }}$ arch artery no NCAM expression was observed (Fig. 2H,L and Fig. 3F,I).

The aortico-pulmonary septum and commissures of the aortic and pulmonary valves, stained more intense with NCAM compared with the wild type embryos and over a larger area (Fig. 4F). In embryos demonstrating dysplastic valves the commissures and wall of the valves became even more positive. The atrioventricular valves and myocardial connection sites, as well as the interventricular septum, did not differ from the wild type embryos (data not shown).

\section{Ductus Venosus Region}

Wild type. From E12 onwards the (sub) endothelial layer of the ductus venosus was slightly NCAM positive (Fig. 5A). This positivity persisted until the connection of the ductus venosus with the inferior caval vein was made. The proximal part of the 

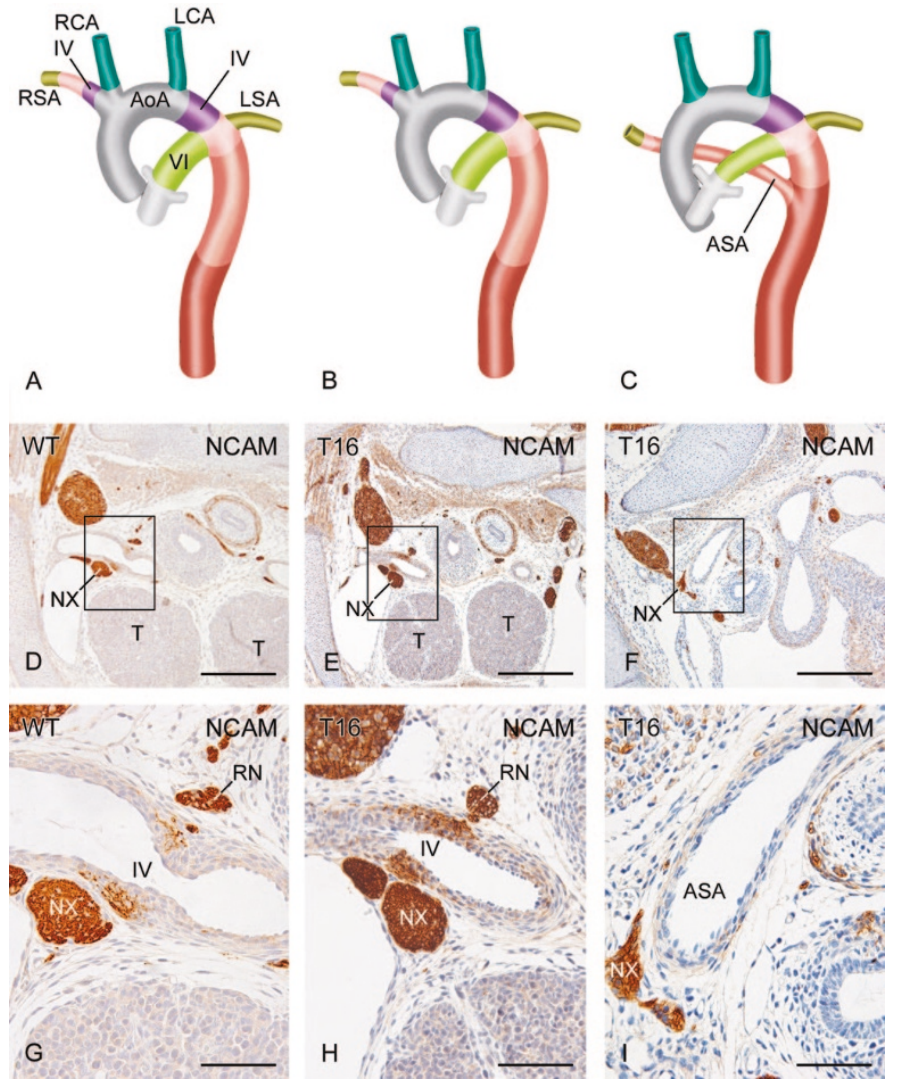

Figure 3. Transverse sections of the aortic arch (AoA) region of wild type (WT) and trisomy 16 (T16) mice at E14; stained for NCAM. (A-C) Schematic outline of normal $(A-B)$ PAA configuration and interrupted right $4^{\text {th }}$ PAA $(C)$. Colors refer to distinct embryonic origins. $(D, G)$ Right $4^{\text {th }}$ PAA with NCAM expression in the adventitia with extension to the media in close proximity to the vagal $(\mathrm{NX})$ and recurrent nerve $(\mathrm{RN}) .(E, H)$ Right $4^{\text {th }}$ PAA of a T16 mouse with a more intense and widespread vascular NCAM expression into the media. $(F, I)$ Aberrant subclavian artery (ASA) and persistent right dorsal aorta in T16 mouse; the right $4^{\text {th }}$ is interrupted an no NCAM can be found in the remaining vascular structures. Abbreviations: RSA/ LSA right/left subclavian artery; RCA/LCA right/ left carotid artery; Bars $=(D-F) 250 \mu \mathrm{m}$ and $(G-\mathrm{I})$ $50 \mu \mathrm{m}$.

ductus venosus, the smooth muscle cell sphincter part of the vessel, was also NCAM positive. The inferior caval vein was negative for NCAM.

Trisomy 16. The vessel wall of the ductus venosus showed a different expression pattern of NCAM as well as altered morphology. The ductus venosus wall was thickened and there was greater NCAM expression in the (sub) endothelium (Fig. $5 B$ ). Similar as in wild type, this positivity persisted until the connection of the ductus venosus with the inferior caval vein was made. The sphincter part of the ducts venosus also showed high NCAM expression. The inferior caval vein was negative for NCAM.

\section{DISCUSSION}

In the present study, trisomy 16 mice embryos were investigated as animal model for the human fetus with increased NT. The hypothesis was tested that a disturbance in neuronal and vascular development is a common denominator in the abnormalities related to increased NT.
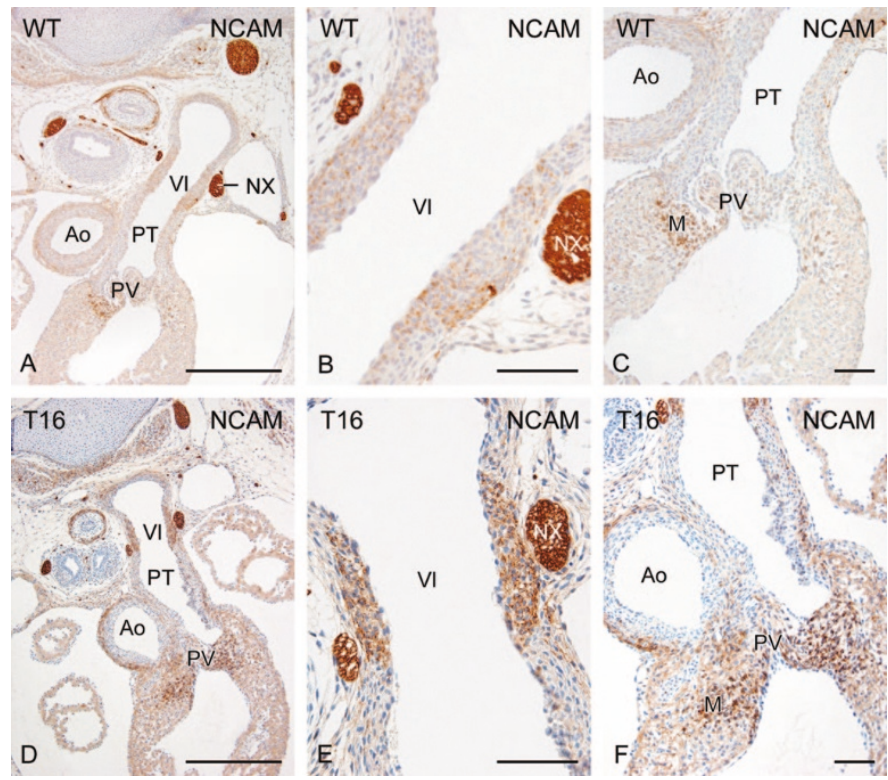

Figure 4. Transverse sections of the outflow tract of wild type (WT) and trisomy 16 (T16) mice at E14 showing $6^{\text {th }}$ PAA (VI), pulmonary trunk (PT) and aorta (Ao). ( $A-B)$ WT mouse with vascular NCAM expression in the vessel wall of the $6^{\text {th }}$ PAA in close proximity of vagal (NX) and recurrent nerve $(\mathrm{RN})$. $(C)$ : The pulmonary valve $(\mathrm{PV})$ is slightly NCAM positive, with more positivity in underlying myocardial cuff $(\mathrm{M})$. The trans section is made just above aortico-pulmonary septum. (D-E) T16 mouse with increased vascular NCAM expression in the vessel wall of $6^{\text {th }}$ PAA compared with WT. $(F)$ Dysplastic PV with high NCAM staining in valve leaflets and underlying myocardial cuff. Bars $=(A, D) 250 \mu \mathrm{m}$ and $(B-C, E-F) 50 \mu \mathrm{m}$.
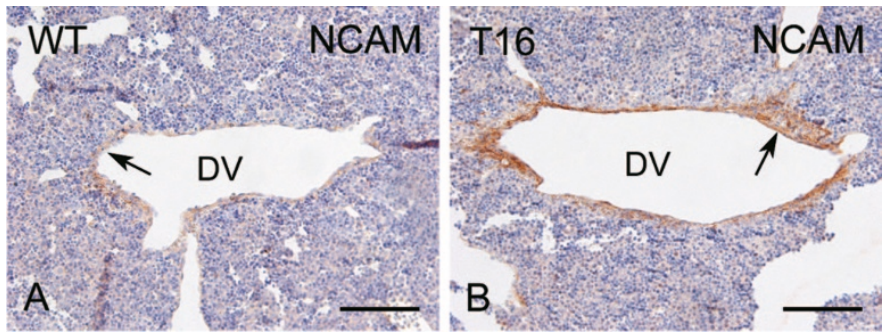

Figure 5. Tranverse sections of the ductus venosus (DV) of wild type (WT) and trisomy 16 (T16) mice. (A) NCAM positivity in (sub)endothelial (arrow) layer of the ductus venosus. (B) Increased NCAM positivity in the (sub)endothelium (arrow) of the ductus venosus, compared with WT. Bars $=(A, B)$ $100 \mu \mathrm{m}$.

Increased NT in the human fetus is associated with trisomy 21 and other aneuploidies, structural defects, mainly cardiovascular defects, enlarged JLS and alterations in the ductus venosus flow velocity (4). Trisomy 16 mice were studied as they have nuchal edema, enlarged JLS and cardiac defects (3) Also, the mouse chromosome 16 contains the syntenic region for the human chromosome band 21q22 (9).

Our study demonstrates an increased NCAM expression in trisomy 16 mouse embryos, which was linked to both neurogenesis and vasculogenesis (Fig. 6). NCAM is a cell adhesion molecule, which affects neural crest cell migration, neuronal fasciculation, neuronal differentiation, and axon guidance $(16,17)$ Interestingly, NCAM is up-regulated by RUNX1, which is expressed on the critical triplicated region of human chromosome 21 and mouse chromosome $16(11,12)$. This 
Model: Trisomy 16 mouse embryo RUNX-1

NCAM-1 upregulation

Neurogenesis
- neuronal differentiation
- axon guidance
- Vagal nerve

Vasculogenesis
- "homing" of neural crest cells
- 4th and 6th PAA
- Ductus venosus

Figure 6. Schematic model of NCAM expression and function in trisomy 16 mice embryos.

could explain the NCAM overexpression seen in the trisomy 16 embryos.

Furthermore, the fact that the NCAM over expression was clearly present in both neck, heart and ductus venosus region was remarkable, because all of these regions are described to be of importance in the pathophysiology of increased NT in the human fetus $(4,18)$. No morphologic alterations linking these regions have been reported before.

The cranial nerves X, IX and XI were smaller in trisomy 16 embryos and the NCAM positivity in the nerve was increased. This indicates that the development of the nerves is disturbed. We postulate that an altered neurogenesis in the cranial nerves could influence the differentiation of the endothelium of the JLS and jugular vein, and cause enlargement of the JLS. Genes of interest involved in such an interaction between nerves and endothelial differentiation are Neuropilin-1 (NP-1) and NP-2. These genes are known to mediate axonal guidance in the developing nervous system, but recently they are also found to play a role in angiogenesis (19).

The abnormal arrangement of the cranial nerves in the neck region of trisomy 16 embryos seems to be caused by the mechanics of the enlarged jugular sac, since abnormal expansion of the JLS displaces the surrounding structures. As a consequence, the abnormal grouping of these nerves might influence the function of the structures, which they innervate.

Furthermore, the vessel wall of the $4^{\text {th }}$ and $6^{\text {th }}$ PAA of the trisomy 16 embryos clearly showed up-regulation of NCAM. In the vessel wall NCAM is most likely involved in vasculogenesis, by the homing of neural crest cells, as has been described before $(20,21)$ NCAM is reported to diminish after neural crest cells have ceased migration (17). Migration of cardiac neural crest cells is needed for normal cardiovascular development. Also, the role of a disturbed migration of neural crest cells in the development of PAA and outflow tract anomalies is generally accepted (22-24). However, as all aortic arch segments contain smooth muscle cells derived from the neural crest there may be a different explanation for the specific increased expression of NCAM in the fourth and sixth arch segment. We postulate a role as homing molecule for the vagal nerve derived neuronal fibers that are specifically present in these vessel segments.

Opposite to the NCAM up-regulation observed in the normal PAA of the trisomy 16 embryos, the defected $4^{\text {th }}$ PAA of the trisomy 16 embryos showed a diminished or lack of NCAM expression. This indicates a disturbed migration of neural crest cells. A relation between a reduced NCAM expression and defected neural crest cell migration has been reported before in the Tgf $\beta 2-/-$ mouse, which is a mouse model for $4^{\text {th }}$ PAA defects. The Tgf $\beta 2-/-$ mouse model also showed a reduced NCAM expression in the similar region in the vessel wall, as in our trisomy 16 embryos (25). Interestingly, TGF $\beta 2$ directly up-regulates NCAM (26). In case of PAA defects, a diminished expression of TGF $\beta 2$ could therefore be involved in a downregulation of NCAM.

Thus, we hypothesize that an increased NCAM expression in trisomy 16 embryos is related to up-regulation by RUNX1, whereas a reduced NCAM expression coincided with PAA defects. The mechanism that leads to a reduced NCAM expression in PAA defects in our model needs further investigation. Nonetheless, the diminished NCAM expression indicates a disturbed neural crest development.

This involvement can also be deduced from the diminished size of the cranial nerves, as the proximal ganglia of cranial nerves IX and $X$ are derived from cardiac neural crest $(27,28)$. A disturbed development and smaller cranial nerves are also found in other models associated with an impaired neural crest development $(29,30)$ Furthermore, perturbation of the neural crest cell development has been proposed in the pathomorphogenesis of several abnormalities associated with Down syndrome; such as cardiac defects and a number of facial and neck abnormalities (27).

In the human fetus with increased NT a variable degree of cardiac defects are seen. The most common defects are septal defects and some fetuses also show minor or major outflow tract and aortic arch anomalies (31). The PAA defects seen in a number of the trisomy 16 mice therefore present the severe end of the phenotype of cardiac defects associated with increased NT in human fetuses.

The up-regulation of NCAM in the vessel wall of the ductus venosus and thickening of this wall, could be related to the changed ductus venosus flow velocities in human fetuses with increased NT, which is present regardless of the existence of a cardiac defect (18). The ductus venosus sphincter contains neural crest derived adrenergic nerves, causing contraction and relaxation, which could influence the blood flow (32). The relation between changes of the ductus venosus flow velocity and innervation of the smooth muscle cell layer of the sphincter part has been suggested earlier (33). The NCAM up-regulation in the ductus venosus was not linked to a specific cardiac morphology in our mouse model. A similar up-regulation in human fetuses could make a morphologic contribution to the alterations in blood flow through the ductus venosus. This is in agreement with former findings altered ductus venosus flow cannot be attributed to altered intracardiac flow nor a specific cardiac morphology in fetuses with increased NT (34).

\section{CONCLUSIONS}

In conclusion, this study shows that trisomy 16 embryos have increased NCAM expression in the neck, heart and ductus venosus region, which was linked to both neuronal and vascu- 
lar development. These findings indicate that a disturbed interaction between neural crest development and endothelial differentiation could be a common denominator in the different anomalies seen in human fetuses with increased NT.

Overexpression of RUNX1 could explain NCAM overexpression in trisomy 16 mice and human fetuses with trisomy 21. Nevertheless, increased NT in the human fetus is also associated with other aneuploidies, such as trisomy 13, trisomy 18 and Turner syndrome, and occurs in cases of normal karyotype (1). It remains to be investigated whether these fetuses also have a disturbed neuronal and vascular development.

We postulate that disturbance of a common developmental process, like neural crest and vascular endothelial development, is responsible for increased NT and associated anomalies. Several genetic origins and environmental influences can lead to a disturbance of this process at different times in development. This could also explain the variety and severity of anomalies in fetuses with nuchal edema.

In the end, morphologic data in human fetuses with increased NT should, therefore, be compared with our findings in the trisomy 16 mouse model.

Acknowledgments. The authors thank Bert Wisse for his technical assistance and Jan Lens for the graphics and layout.

\section{REFERENCES}

1. Snijders RJ, Noble P, Sebire N, Souka A, Nicolaides KH 1998 UK multicentre project on assessment of risk of trisomy 21 by maternal age and fetal nuchal-translucency thickness at 10-14 weeks of gestation. Lancet 352:343-346

2. Matias A, Huggon I, Areias JC, Montenegro N, Nicolaides KH 1999 Cardiac defects in chromosomally normal fetuses with abnormal ductus venosus blood flow at 10-14 weeks. Ultrasound Obstet Gynecol 14:307-310

3. Gittenberger-De Groot AC, Van Den Akker NM, Bartelings MM, Webb S, van Vugt JM, Haak MC 2004 Abnormal lymphatic development in trisomy 16 mouse embryos precedes nuchal edema. Dev Dyn 230:378-384

4. Haak MC, Bartelings MM, Jackson DG, Webb S, Van Vugt JM, Gittenberger-de Groot AC 2002 Increased nuchal translucency is associated with jugular lymphatic distension. Hum Reprod 17:1086-1092

5. Hyett J, Moscoso G, Nicolaides K 1997 Abnormalities of the heart and great arteries in first trimester chromosomally abnormal fetuses. Am J Med Genet 69:207-216

6. Montenegro N, Matias A, Areias JC, Castedo S, Barros H 1997 Increased fetal nuchal translucency: possible involvement of early cardiac failure. Ultrasound Obstet Gynecol 10:265-268

7. von Kaisenberg CS, Prols F, Nicolaides KH, Maass N, Meinhold-Heerlein I, BrandSaberi B 2003 Glycosaminoglycans and proteoglycans in the skin of aneuploid fetuses with increased nuchal translucency. Hum Reprod 18:2544-2561

8. Carmeliet P 2003 Blood vessels and nerves: common signals, pathways and diseases. Nat Rev Genet 4:710-720

9. Miyabara S, Gropp A, Winking H 1982 Trisomy 16 in the mouse fetus associated with generalized edema and cardiovascular and urinary tract anomalies. Teratology 25:369-380

10. Wharton J, Gordon L, Walsh FS, Flanigan TP, Moore SE, Polak JM 1989 Neural cel adhesion molecule (N-CAM) expression during cardiac development in the rat. Brain Res 483:170-176

11. Gattenlohner S, Waller C, Ertl G, Bultmann BD, Muller-Hermelink HK, Marx A $2003 \mathrm{NCAM}(\mathrm{CD} 56)$ and RUNX1(AML1) are up-regulated in human ischemic cardiomyopathy and a rat model of chronic cardiac ischemia. Am J Pathol 163:10811090

12. Lyle R, Gehrig C, Neergaard-Henrichsen C, Deutsch S, Antonarakis SE 2004 Gene expression from the aneuploid chromosome in a trisomy mouse model of down syndrome. Genome Res 14:1268-1274

13. Webb S, Browne NA, Anderson RH 1997 Cardiac morphology at late fetal stages in the mouse with trisomy 16: consequences for different formation of the atrioventricular junction when compared to humans with trisomy 21. Cardiovasc Res 34:515-524

14. Bouman HG, Broekhuizen LA, Baasten AM, Gittenberger-de-Groot AC, Wenink AC 1997 Stereological study of stage 34 chicken hearts with looping disturbances after retinoic acid treatment: disturbed growth of myocardium and atrioventricular cushion tissue. Anat Rec 248:242-250

15. Gundersen HJ, Jensen EB 1987 The efficiency of systematic sampling in stereology and its prediction. J Microsc 147:229-263

16. Walsh FS, Doherty P 1997 Neural cell adhesion molecules of the immunoglobulin superfamily: role in axon growth and guidance. Annu Rev Cell Dev Biol 13:425-456

17. Akitaya T, Bronner-Fraser M 1992 Expression of cell adhesion molecules during initiation and cessation of neural crest cell migration. Dev Dyn 194:12-20

18. Haak MC, Twisk JW, Bartelings MM, Gittenberger-de Groot AC, Van Vugt, JM 2003 Ductus venosus flow velocities in relation to the cardiac defects in first-trimeste fetuses with enlarged nuchal translucency. Am J Obstet Gynecol 188:727-733

19. Yamada Y, Takakura N, Yasue H, Ogawa H, Fujisawa H, Suda T 2001 Exogenous clustered neuropilin 1 enhances vasculogenesis and angiogenesis. Blood 97:16711678

20. Nishijima S, Nakagawa M, Fujino H, Hanato T, Okamoto N, Shimada M 2000 Teratogenic effects of bis-diamine on early embryonic rat heart: an in vitro study. Teratology 62:115-122

21. Okagawa H, Nakagawa M, Simada M 1995 Immunolocalization of N-CAM in the heart of the early developing rat embryo. Anat Rec 243:261-271

22. Waller BR, McQuinn T, Phelps AL, Markwald RR, Lo CW, Thompson RP, Wessels A 2000 Conotruncal anomalies in the trisomy 16 mouse: an immunohistochemical analysis with emphasis on the involvement of the neural crest. Anat Rec 260:279-293

23. Bergwerff M, DeRuiter MC, Hall S, Poelmann RE, Gittenberger-De Groot AC 1999 Unique vascular morphology of the fourth aortic arches: possible implications for pathogenesis of type-B aortic arch interruption and anomalous right subclavian artery. Cardiovasc Res 44:185-196

24. Boot MJ, Gittenberger-De Groot AC, Van Iperen, L, Hierck BP, Poelmann RE 2003 Spatiotemporally separated cardiac neural crest subpopulations that target the outflow tract septum and pharyngeal arch arteries. Anat Rec A Discov Mol Cell Evol Biol 275:1009-1018

25. Molin DG, Poelmann RE, DeRuiter MC, Azhar M, Doetschman T, Gittenberger-deGroot AC 2004 Transforming growth factor beta-SMAD2 signaling regulates aortic arch innervation and development. Circ Res 95:1109-1117

26. Roubin R, Deagostini-Bazin H, Hirsch MR, Goridis C 1990 Modulation of NCAM expression by transforming growth factor-beta, serum and autocrine factors. J Cell Biol 111:673-684

27. Kirby ML 1991 Neural crest and the morphogenesis of Down syndrome with special emphasis on cardiovascular development. Prog Clin Biol Res 373:215225

28. Kuratani SC, Miyagawa-Tomita S, Kirby ML 1991 Development of cranial nerves in the chick embryo with special reference to the alterations of cardiac branches after ablation of the cardiac neural crest. Anat Embryol 183:501-514

29. Van Maele-Fabry G, Gofflot F, Clotman F, Picard JJ 1995 Alterations of mouse embryonic branchial nerves and ganglia induced by ethanol. Neurotox Terato 17:497-506

30. Cederberg J, Picard JJ, Eriksson UJ 2003 Maternal diabetes in the rat impairs the formation of neural-crest derived cranial nerve ganglia in the offspring. Diabetolologia 46:1245-1251

31. Hyett J, Moscoso G, Papapanagiotou G, Perdu M, Nicolaides KH 1996 Abnormalities of the heart and great arteries in chromosomally normal fetuses with increased nuchal translucency thickness at 11-13 weeks of gestation. Ultrasound Obstet Gynecol $7: 245-250$

32. Coceani F, Adeagbo AS, Cutz E, Olley PM 1984 Autonomic mechanisms in the ductus venosus of the lamb. Am J Physiol 247:H17-H24

33. Mavrides E, Moscoso G, Carvalho JS, Campbell S, Thilaganathan B 2002 The human ductus venosus between 13 and 17 weeks of gestation: histological and morphometric studies. Ultrasound Obstet Gynecol 19:39-46

34. Haak MC, Twisk JW, Bartelings MM, Gittenberger-De Groot AC, van Vugt, JM 2005 First-trimester fetuses with increased nuchal translucency do not show altered intracardiac flow velocities. Ultrasound Obstet Gynecol 25:246-252 